\title{
Integral of Non Positive Functions
}

\author{
Noboru Endou \\ National Institute of Technology, Gifu College \\ 2236-2 Kamimakuwa, Motosu, Gifu, Japan
}

\begin{abstract}
Summary. In this article, we formalize in the Mizar system [1, 7] the Lebesgue type integral and convergence theorems for non positive functions [8], [2]. Many theorems are based on our previous results [5], 6].
\end{abstract}

MSC: 28A25 03B35

Keywords: integration of non positive function

MML identifier: MESFUN11, version: 8.1.06 5.44.1305

\section{Preliminaries}

Let $X$ be a non empty set and $f$ be a non-negative partial function from $X$ to $\overline{\mathbb{R}}$. Observe that $-f$ is non-positive.

Let $f$ be a non-positive partial function from $X$ to $\overline{\mathbb{R}}$. One can check that $-f$ is non-negative.

Now we state the propositions:

(1) Let us consider a non empty set $X$, a non-positive partial function $f$ from $X$ to $\overline{\mathbb{R}}$, and a set $E$. Then $f \nmid E$ is non-positive.

(2) Let us consider a non empty set $X$, a set $A$, a real number $r$, and a partial function $f$ from $X$ to $\overline{\mathbb{R}}$. Then $(r \cdot f)\lceil A=r \cdot(f\lceil A)$.

(3) Let us consider a non empty set $X$, a set $A$, and a partial function $f$ from $X$ to $\overline{\mathbb{R}}$. Then $-f\lceil A=(-f)\lceil A$. The theorem is a consequence of (2).

(4) Let us consider a non empty set $X$, a partial function $f$ from $X$ to $\overline{\mathbb{R}}$, and a real number $c$. Suppose $f$ is non-positive. Then

(i) if $0 \leqslant c$, then $c \cdot f$ is non-positive, and

(ii) if $c \leqslant 0$, then $c \cdot f$ is non-negative. 
(5) Let us consider a non empty set $X$, a $\sigma$-field $S$ of subsets of $X$, a $\sigma$ measure $M$ on $S$, and a partial function $f$ from $X$ to $\overline{\mathbb{R}}$. Then

(i) $\max _{+}(f)$ is non-negative, and

(ii) $\max _{-}(f)$ is non-negative, and

(iii) $|f|$ is non-negative.

(6) Let us consider a non empty set $X$, a partial function $f$ from $X$ to $\overline{\mathbb{R}}$, and an object $x$. Then

(i) $f(x) \leqslant\left(\max _{+}(f)\right)(x)$, and

(ii) $f(x) \geqslant-\left(\max _{-}(f)\right)(x)$.

(7) Let us consider a non empty set $X$, a partial function $f$ from $X$ to $\overline{\mathbb{R}}$, and a positive real number $r$. Then LE-dom $(f, r)=\mathrm{LE}-\operatorname{dom}\left(\max _{+}(f), r\right)$.

(8) Let us consider a non empty set $X$, a partial function $f$ from $X$ to $\overline{\mathbb{R}}$, and a non positive real number $r$. Then LE-dom $(f, r)=\mathrm{GT}-\operatorname{dom}\left(\max _{-}(f),-r\right)$.

(9) Let us consider a non empty set $X$, partial functions $f, g$ from $X$ to $\overline{\mathbb{R}}$, an extended real $a$, and a real number $r$. Suppose $r \neq 0$ and $g=r \cdot f$. Then EQ-dom $(f, a)=\mathrm{EQ}-\operatorname{dom}(g, a \cdot r)$.

(10) Let us consider a non empty set $X$, a $\sigma$-field $S$ of subsets of $X$, a partial function $f$ from $X$ to $\overline{\mathbb{R}}$, and an element $A$ of $S$. Suppose $A \subseteq \operatorname{dom} f$. Then $f$ is measurable on $A$ if and only if $\max _{+}(f)$ is measurable on $A$ and $\max _{-}(f)$ is measurable on $A$.

Let $X$ be a non empty set, $f$ be a function from $X$ into $\overline{\mathbb{R}}$, and $r$ be a real number. Note that the functor $r \cdot f$ yields a function from $X$ into $\overline{\mathbb{R}}$. Now we state the proposition:

(11) Let us consider a non empty set $X$, a real number $r$, and a without $+\infty$ function $f$ from $X$ into $\overline{\mathbb{R}}$. If $r \geqslant 0$, then $r \cdot f$ is without $+\infty$.

Let $X$ be a non empty set, $f$ be a without $+\infty$ function from $X$ into $\overline{\mathbb{R}}$, and $r$ be a non negative real number. Let us note that $r \cdot f$ is without $+\infty$ as a function from $X$ into $\overline{\mathbb{R}}$.

Now we state the proposition:

(12) Let us consider a non empty set $X$, a real number $r$, and a without $+\infty$ function $f$ from $X$ into $\overline{\mathbb{R}}$. If $r \leqslant 0$, then $r \cdot f$ is without $-\infty$.

Let $X$ be a non empty set, $f$ be a without $+\infty$ function from $X$ into $\overline{\mathbb{R}}$, and $r$ be a non positive real number. One can check that $r \cdot f$ is without $-\infty$.

Now we state the proposition:

(13) Let us consider a non empty set $X$, a real number $r$, and a without $-\infty$ function $f$ from $X$ into $\overline{\mathbb{R}}$. If $r \geqslant 0$, then $r \cdot f$ is without $-\infty$. 
Let $X$ be a non empty set, $f$ be a without $-\infty$ function from $X$ into $\overline{\mathbb{R}}$, and $r$ be a non negative real number. One can check that $r \cdot f$ is without $-\infty$.

Now we state the proposition:

(14) Let us consider a non empty set $X$, a real number $r$, and a without $-\infty$ function $f$ from $X$ into $\overline{\mathbb{R}}$. If $r \leqslant 0$, then $r \cdot f$ is without $+\infty$.

Let $X$ be a non empty set, $f$ be a without $-\infty$ function from $X$ into $\overline{\mathbb{R}}$, and $r$ be a non positive real number. One can check that $r \cdot f$ is without $+\infty$.

Now we state the proposition:

(15) Let us consider a non empty set $X$, a real number $r$, and a without $-\infty$, without $+\infty$ function $f$ from $X$ into $\overline{\mathbb{R}}$. Then $r \cdot f$ is without $-\infty$ and without $+\infty$.

Let $X$ be a non empty set, $f$ be a without $-\infty$, without $+\infty$ function from $X$ into $\overline{\mathbb{R}}$, and $r$ be a real number. Note that $r \cdot f$ is without $-\infty$ and without $+\infty$.

Now we state the propositions:

(16) Let us consider a non empty set $X$, a positive real number $r$, and a function $f$ from $X$ into $\overline{\mathbb{R}}$. Then $f$ is without $+\infty$ if and only if $r \cdot f$ is without $+\infty$.

(17) Let us consider a non empty set $X$, a negative real number $r$, and a function $f$ from $X$ into $\overline{\mathbb{R}}$. Then $f$ is without $+\infty$ if and only if $r \cdot f$ is without $-\infty$.

(18) Let us consider a non empty set $X$, a positive real number $r$, and a function $f$ from $X$ into $\overline{\mathbb{R}}$. Then $f$ is without $-\infty$ if and only if $r \cdot f$ is without $-\infty$.

(19) Let us consider a non empty set $X$, a negative real number $r$, and a function $f$ from $X$ into $\overline{\mathbb{R}}$. Then $f$ is without $-\infty$ if and only if $r \cdot f$ is without $+\infty$.

(20) Let us consider a non empty set $X$, a non zero real number $r$, and a function $f$ from $X$ into $\overline{\mathbb{R}}$. Then $f$ is without $-\infty$ and without $+\infty$ if and only if $r \cdot f$ is without $-\infty$ and without $+\infty$. The theorem is a consequence of (16), (18), (17), and (19).

(21) Let us consider non empty sets $X, Y$, a partial function $f$ from $X$ to $\overline{\mathbb{R}}$, and a real number $r$. Suppose $f=Y \longmapsto r$. Then $f$ is without $-\infty$ and without $+\infty$.

(22) Let us consider a non empty set $X$, and a function $f$ from $X$ into $\overline{\mathbb{R}}$. Then

(i) $0 \cdot f=X \longmapsto 0$, and

(ii) $0 \cdot f$ is without $-\infty$ and without $+\infty$. 
Proof: For every element $x$ of $X,(0 \cdot f)(x)=(X \longmapsto 0)(x)$.

(23) Let us consider a non empty set $X$, and partial functions $f, g$ from $X$ to $\overline{\mathbb{R}}$. Suppose $f$ is without $-\infty$ and without $+\infty$. Then

(i) $\operatorname{dom}(f+g)=\operatorname{dom} f \cap \operatorname{dom} g$, and

(ii) $\operatorname{dom}(f-g)=\operatorname{dom} f \cap \operatorname{dom} g$, and

(iii) $\operatorname{dom}(g-f)=\operatorname{dom} f \cap \operatorname{dom} g$.

Let us consider a non empty set $X$ and functions $f_{1}, f_{2}$ from $X$ into $\overline{\mathbb{R}}$. Now we state the propositions:

(24) Suppose $f_{2}$ is without $-\infty$ and without $+\infty$. Then

(i) $f_{1}+f_{2}$ is a function from $X$ into $\overline{\mathbb{R}}$, and

(ii) for every element $x$ of $X,\left(f_{1}+f_{2}\right)(x)=f_{1}(x)+f_{2}(x)$.

The theorem is a consequence of (23).

(25) Suppose $f_{1}$ is without $-\infty$ and without $+\infty$. Then

(i) $f_{1}-f_{2}$ is a function from $X$ into $\overline{\mathbb{R}}$, and

(ii) for every element $x$ of $X,\left(f_{1}-f_{2}\right)(x)=f_{1}(x)-f_{2}(x)$.

The theorem is a consequence of (23).

(26) Suppose $f_{2}$ is without $-\infty$ and without $+\infty$. Then

(i) $f_{1}-f_{2}$ is a function from $X$ into $\overline{\mathbb{R}}$, and

(ii) for every element $x$ of $X,\left(f_{1}-f_{2}\right)(x)=f_{1}(x)-f_{2}(x)$.

The theorem is a consequence of (23).

(27) Let us consider non empty sets $X, Y$, and partial functions $f_{1}, f_{2}$ from $X$ to $\overline{\mathbb{R}}$. Suppose $\operatorname{dom} f_{1} \subseteq Y$ and $f_{2}=Y \longmapsto 0$. Then

(i) $f_{1}+f_{2}=f_{1}$, and

(ii) $f_{1}-f_{2}=f_{1}$, and

(iii) $f_{2}-f_{1}=-f_{1}$.

The theorem is a consequence of (21) and (23).

Let us consider a non empty set $X$, a $\sigma$-field $S$ of subsets of $X$, a $\sigma$-measure $M$ on $S$, and partial functions $f, g$ from $X$ to $\overline{\mathbb{R}}$. Now we state the propositions:

(28) If $f$ is simple function in $S$ and $g$ is simple function in $S$, then $f+g$ is simple function in $S$.

Proof: Consider $F$ being a finite sequence of separated subsets of $S, a$ being a finite sequence of elements of $\overline{\mathbb{R}}$ such that $F$ and $a$ are representation of $f$. Consider $G$ being a finite sequence of separated subsets of $S$, $b$ being a finite sequence of elements of $\overline{\mathbb{R}}$ such that $G$ and $b$ are representation of $g$. Set $l_{1}=$ len $a$. Set $l_{2}=$ len $b$. Define $\mathcal{H}$ (natural number $)=$ 
$F\left(\left(\$_{1}-^{\prime} 1 \operatorname{div} l_{2}\right)+1\right) \cap G\left(\left(\$_{1}-^{\prime} 1 \bmod l_{2}\right)+1\right)$. Consider $F_{1}$ being a finite sequence such that len $F_{1}=l_{1} \cdot l_{2}$ and for every natural number $k$ such that $k \in \operatorname{dom} F_{1}$ holds $F_{1}(k)=\mathcal{H}(k)$. For every natural numbers $k, l$ such that $k, l \in \operatorname{dom} F_{1}$ and $k \neq l$ holds $F_{1}(k)$ misses $F_{1}(l) \cdot \operatorname{dom}(f+g)=\bigcup \operatorname{rng} F_{1}$. For every natural number $k$ and for every elements $x, y$ of $X$ such that $k \in \operatorname{dom} F_{1}$ and $x, y \in F_{1}(k)$ holds $(f+g)(x)=(f+g)(y)$.

(29) If $f$ is simple function in $S$ and $g$ is simple function in $S$, then $f-g$ is simple function in $S$. The theorem is a consequence of (28).

(30) Let us consider a non empty set $X$, a $\sigma$-field $S$ of subsets of $X$, and a partial function $f$ from $X$ to $\overline{\mathbb{R}}$. If $f$ is simple function in $S$, then $-f$ is simple function in $S$.

(31) Let us consider a non empty set $X$, and a non-negative partial function $f$ from $X$ to $\overline{\mathbb{R}}$. Then $f=\max _{+}(f)$.

Proof: For every element $x$ of $X$ such that $x \in \operatorname{dom} f$ holds $f(x)=$ $\left(\max _{+}(f)\right)(x)$.

(32) Let us consider a non empty set $X$, and a non-positive partial function $f$ from $X$ to $\overline{\mathbb{R}}$. Then $f=-\max _{-}(f)$.

Proof: For every element $x$ of $X$ such that $x \in \operatorname{dom} f$ holds $f(x)=$ $\left(-\max _{-}(f)\right)(x)$.

(33) Let us consider a non empty set $C$, a partial function $f$ from $C$ to $\overline{\mathbb{R}}$, and a real number $c$. Suppose $c \leqslant 0$. Then

(i) $\max _{+}(c \cdot f)=(-c) \cdot \max _{-}(f)$, and

(ii) $\max _{-}(c \cdot f)=(-c) \cdot \max _{+}(f)$.

Proof: For every element $x$ of $C$ such that $x \in \operatorname{dom}_{\max }(c \cdot f)$ holds $\left(\max _{+}(c \cdot f)\right)(x)=\left((-c) \cdot \max _{-}(f)\right)(x)$. For every element $x$ of $C$ such that $x \in$ dom $\max _{-}(c \cdot f)$ holds $\left(\max _{-}(c \cdot f)\right)(x)=\left((-c) \cdot \max _{+}(f)\right)(x)$.

(34) Let us consider a non empty set $X$, and a partial function $f$ from $X$ to $\overline{\mathbb{R}}$. Then $\max _{+}(f)=\max _{-}(-f)$. The theorem is a consequence of $(33)$.

(35) Let us consider a non empty set $X$, a partial function $f$ from $X$ to $\overline{\mathbb{R}}$, and real numbers $r_{1}, r_{2}$. Then $r_{1} \cdot\left(r_{2} \cdot f\right)=\left(r_{1} \cdot r_{2}\right) \cdot f$.

(36) Let us consider a non empty set $X$, and partial functions $f, g$ from $X$ to $\overline{\mathbb{R}}$. If $f=-g$, then $g=-f$. The theorem is a consequence of $(35)$.

Let $X$ be a non empty set, $F$ be a sequence of partial functions from $X$ into $\overline{\mathbb{R}}$, and $r$ be a real number. The functor $r \cdot F$ yielding a sequence of partial functions from $X$ into $\overline{\mathbb{R}}$ is defined by

(Def. 1) for every natural number $n$, it $(n)=r \cdot F(n)$.

The functor $-F$ yielding a sequence of partial functions from $X$ into $\overline{\mathbb{R}}$ is defined by the term 
(Def. 2) $(-1) \cdot F$.

Now we state the proposition:

(37) Let us consider a non empty set $X$, a sequence $F$ of partial functions from $X$ into $\overline{\mathbb{R}}$, and a natural number $n$. Then $(-F)(n)=-F(n)$.

Let us consider a non empty set $X$, a sequence $F$ of partial functions from $X$ into $\overline{\mathbb{R}}$, and an element $x$ of $X$. Now we state the propositions:

(38) $(-F) \# x=-F \# x$. The theorem is a consequence of (37).

(39) (i) $F \# x$ is convergent to $+\infty$ iff $(-F) \# x$ is convergent to $-\infty$, and

(ii) $F \# x$ is convergent to $-\infty$ iff $(-F) \# x$ is convergent to $+\infty$, and

(iii) $F \# x$ is convergent to a finite limit iff $(-F) \# x$ is convergent to a finite limit, and

(iv) $F \# x$ is convergent iff $(-F) \# x$ is convergent, and

(v) if $F \# x$ is convergent, then $\lim ((-F) \# x)=-\lim (F \# x)$.

The theorem is a consequence of (38).

Let us consider a non empty set $X$ and a sequence $F$ of partial functions from $X$ into $\overline{\mathbb{R}}$. Now we state the propositions:

(40) If $F$ has the same dom, then $-F$ has the same dom. The theorem is a consequence of (37).

(41) If $F$ is additive, then $-F$ is additive. The theorem is a consequence of $(37)$.

(42) Let us consider a non empty set $X$, a sequence $F$ of partial functions from $X$ into $\overline{\mathbb{R}}$, and a natural number $n$. Then $\left(\sum_{\alpha=0}^{\kappa}(-F)(\alpha)\right)_{\kappa \in \mathbb{N}}(n)=$ $\left(-\left(\sum_{\alpha=0}^{\kappa} F(\alpha)\right)_{\kappa \in \mathbb{N}}\right)(n)$.

Proof: Define $\mathcal{P}$ [natural number $] \equiv\left(\sum_{\alpha=0}^{\kappa}(-F)(\alpha)\right)_{\kappa \in \mathbb{N}}\left(\$_{1}\right)=$ $\left(-\left(\sum_{\alpha=0}^{\kappa} F(\alpha)\right)_{\kappa \in \mathbb{N}}\right)\left(\$_{1}\right) . \mathcal{P}[0]$. For every natural number $k$ such that $\mathcal{P}[k]$ holds $\mathcal{P}[k+1]$. For every natural number $k, \mathcal{P}[k]$.

(43) Let us consider a sequence $s$ of extended reals, and a natural number $n$. Then $\left(\sum_{\alpha=0}^{\kappa}(-s)(\alpha)\right)_{\kappa \in \mathbb{N}}(n)=-\left(\sum_{\alpha=0}^{\kappa} s(\alpha)\right)_{\kappa \in \mathbb{N}}(n)$.

Proof: Define $\mathcal{P}$ [natural number] $\equiv\left(\sum_{\alpha=0}^{\kappa}(-s)(\alpha)\right)_{\kappa \in \mathbb{N}}\left(\$_{1}\right)=$ $-\left(\sum_{\alpha=0}^{\kappa} s(\alpha)\right)_{\kappa \in \mathbb{N}}\left(\$_{1}\right)$. For every natural number $k$ such that $\mathcal{P}[k]$ holds $\mathcal{P}[k+1]$. For every natural number $k, \mathcal{P}[k]$.

Let us consider a sequence $s$ of extended reals. Now we state the propositions:

(44) $\left(\sum_{\alpha=0}^{\kappa}(-s)(\alpha)\right)_{\kappa \in \mathbb{N}}=-\left(\sum_{\alpha=0}^{\kappa} s(\alpha)\right)_{\kappa \in \mathbb{N}}$. The theorem is a consequence of $(43)$.

(45) If $s$ is summable, then $-s$ is summable. The theorem is a consequence of (44). 
Let us consider a non empty set $X$ and a sequence $F$ of partial functions from $X$ into $\overline{\mathbb{R}}$. Now we state the propositions:

(46) If for every natural number $n, F(n)$ is without $+\infty$, then $F$ is additive.

(47) If for every natural number $n, F(n)$ is without $-\infty$, then $F$ is additive.

(48) Let us consider a non empty set $X$, a sequence $F$ of partial functions from $X$ into $\overline{\mathbb{R}}$, and an element $x$ of $X$. Suppose $F \# x$ is summable. Then

(i) $(-F) \# x$ is summable, and

(ii) $\sum((-F) \# x)=-\sum(F \# x)$.

The theorem is a consequence of (45), (38), and (44).

(49) Let us consider a non empty set $X$, a $\sigma$-field $S$ of subsets of $X$, and a sequence $F$ of partial functions from $X$ into $\overline{\mathbb{R}}$. Suppose $F$ is additive and has the same dom and for every element $x$ of $X$ such that $x \in \operatorname{dom}(F(0))$ holds $F \# x$ is summable. Then $\lim \left(\sum_{\alpha=0}^{\kappa}(-F)(\alpha)\right)_{\kappa \in \mathbb{N}}=-\lim \left(\sum_{\alpha=0}^{\kappa} F(\alpha)\right)_{\kappa \in \mathbb{N}}$. Proof: Set $G=-F$. For every element $n$ of $\mathbb{N},\left(\sum_{\alpha=0}^{\kappa} G(\alpha)\right)_{\kappa \in \mathbb{N}}(n)=$ $\left(-\left(\sum_{\alpha=0}^{\kappa} F(\alpha)\right)_{\kappa \in \mathbb{N}}\right)(n)$. For every element $x$ of $X$ such that $x \in \operatorname{dom} \lim$ $\left(\sum_{\alpha=0}^{\kappa} G(\alpha)\right)_{\kappa \in \mathbb{N}}$ holds $\left(\lim \left(\sum_{\alpha=0}^{\kappa} G(\alpha)\right)_{\kappa \in \mathbb{N}}\right)(x)=$ $\left(-\lim \left(\sum_{\alpha=0}^{\kappa} F(\alpha)\right)_{\kappa \in \mathbb{N}}\right)(x)$.

(50) Let us consider a non empty set $X$, a $\sigma$-field $S$ of subsets of $X$, sequences $F, G$ of partial functions from $X$ into $\overline{\mathbb{R}}$, and an element $E$ of $S$. Suppose $E \subseteq \operatorname{dom}(F(0))$ and $F$ is additive and has the same dom and for every natural number $n, G(n)=F(n)\left\lceil E\right.$. Then $\lim \left(\sum_{\alpha=0}^{\kappa} G(\alpha)\right)_{\kappa \in \mathbb{N}}=$ $\lim \left(\sum_{\alpha=0}^{\kappa} F(\alpha)\right)_{\kappa \in \mathbb{N}}\lceil E$.

Proof: For every element $x$ of $X$ such that $x \in E$ holds $F \# x=G \# x$. Set $P_{1}=\left(\sum_{\alpha=0}^{\kappa} F(\alpha)\right)_{\kappa \in \mathbb{N}}$. Set $P_{2}=\left(\sum_{\alpha=0}^{\kappa} G(\alpha)\right)_{\kappa \in \mathbb{N}}$. For every element $x$ of $X$ such that $x \in \operatorname{dom} \lim P_{2}$ holds $\left(\lim P_{2}\right)(x)=\left(\lim P_{1}\right)(x)$. For every element $x$ of $X$ such that $x \in \operatorname{dom}\left(\lim P_{2}\lceil E)\right.$ holds $\left(\lim P_{2}\lceil E)(x)=\right.$ $\left(\lim P_{1}\lceil E)(x)\right.$.

\section{Integral of Non Positive Measurable Functions}

Now we state the propositions:

(51) Let us consider a non empty set $X$, a $\sigma$-field $S$ of subsets of $X$, a $\sigma$ measure $M$ on $S$, and a non-negative partial function $f$ from $X$ to $\overline{\mathbb{R}}$. Then $\int^{\prime} \max _{-}(-f) \mathrm{d} M=\int^{\prime} f \mathrm{~d} M$. The theorem is a consequence of (32), (36), and (35).

(52) Let us consider a non empty set $X$, a $\sigma$-field $S$ of subsets of $X$, a $\sigma$ measure $M$ on $S$, a partial function $f$ from $X$ to $\overline{\mathbb{R}}$, and an element $A$ of $S$. 
Suppose $A=\operatorname{dom} f$ and $f$ is measurable on $A$. Then $\int-f \mathrm{~d} M=-\int f \mathrm{~d} M$. The theorem is a consequence of (36), (10), (5), and (34).

(53) Let us consider a non empty set $X$, a $\sigma$-field $S$ of subsets of $X$, a $\sigma$ measure $M$ on $S$, a non-negative partial function $f$ from $X$ to $\overline{\mathbb{R}}$, and an element $E$ of $S$. Suppose $E=\operatorname{dom} f$ and $f$ is measurable on $E$. Then

(i) $\int \max _{-}(f) \mathrm{d} M=0$, and

(ii) $\int^{+} \max _{-}(f) \mathrm{d} M=0$.

Proof: $\max _{-}(f)$ is measurable on $E$. For every object $x$ such that $x \in$ dom $\max _{-}(f)$ holds $\left(\max _{-}(f)\right)(x)=0$.

Let us consider a non empty set $X$, a $\sigma$-field $S$ of subsets of $X$, a $\sigma$-measure $M$ on $S$, a partial function $f$ from $X$ to $\overline{\mathbb{R}}$, and an element $E$ of $S$. Now we state the propositions:

(54) If $E=\operatorname{dom} f$ and $f$ is measurable on $E$, then $\int f \mathrm{~d} M=\int \max _{+}(f) \mathrm{d} M-$ $\int \max _{-}(f) \mathrm{d} M$. The theorem is a consequence of (10) and (5).

(55) If $E \subseteq \operatorname{dom} f$ and $f$ is measurable on $E$, then $\int(-f)\left\lceil E \mathrm{~d} M=-\int f\lceil E \mathrm{~d} M\right.$. The theorem is a consequence of (3) and (52).

(56) Let us consider a non empty set $X$, a $\sigma$-field $S$ of subsets of $X$, and a partial function $f$ from $X$ to $\overline{\mathbb{R}}$. Suppose there exists an element $A$ of $S$ such that $A=\operatorname{dom} f$ and $f$ is measurable on $A$ and ( $f$ qua extended real-valued function) is non-positive. Then there exists a sequence $F$ of partial functions from $X$ into $\overline{\mathbb{R}}$ such that

(i) for every natural number $n, F(n)$ is simple function in $S$ and $\operatorname{dom}(F(n))=\operatorname{dom} f$, and

(ii) for every natural number $n, F(n)$ is non-positive, and

(iii) for every natural numbers $n, m$ such that $n \leqslant m$ for every element $x$ of $X$ such that $x \in \operatorname{dom} f$ holds $F(n)(x) \geqslant F(m)(x)$, and

(iv) for every element $x$ of $X$ such that $x \in \operatorname{dom} f$ holds $F \# x$ is convergent and $\lim (F \# x)=f(x)$.

The theorem is a consequence of (37), (30), and (39).

(57) Let us consider a non empty set $X$, a $\sigma$-field $S$ of subsets of $X$, a $\sigma$ measure $M$ on $S$, an element $E$ of $S$, and a non-positive partial function $f$ from $X$ to $\overline{\mathbb{R}}$. Suppose there exists an element $A$ of $S$ such that $A=\operatorname{dom} f$ and $f$ is measurable on $A$. Then

(i) $\int f \mathrm{~d} M=-\int^{+} \max _{-}(f) \mathrm{d} M$, and

(ii) $\int f \mathrm{~d} M=-\int^{+}-f \mathrm{~d} M$, and

(iii) $\int f \mathrm{~d} M=-\int-f \mathrm{~d} M$. 
Proof: Consider $A$ being an element of $S$ such that $A=\operatorname{dom} f$ and $f$ is measurable on $A . f=-\max _{-}(f) .-f=\max _{-}(f)$. For every element $x$ of $X$ such that $x \in \operatorname{dom} \max _{+}(f)$ holds $\left(\max _{+}(f)\right)(x)=0$.

(58) Let us consider a non empty set $X$, a $\sigma$-field $S$ of subsets of $X$, a $\sigma$ measure $M$ on $S$, and a non-positive partial function $f$ from $X$ to $\overline{\mathbb{R}}$. Suppose $f$ is simple function in $S$. Then

(i) $\int f \mathrm{~d} M=-\int^{\prime}-f \mathrm{~d} M$, and

(ii) $\int f \mathrm{~d} M=-\int^{\prime} \max _{-}(f) \mathrm{d} M$.

The theorem is a consequence of (30), (57), (32), and (36).

Let us consider a non empty set $X$, a $\sigma$-field $S$ of subsets of $X$, a $\sigma$-measure $M$ on $S$, a partial function $f$ from $X$ to $\overline{\mathbb{R}}$, and a real number $c$. Now we state the propositions:

(59) If $f$ is simple function in $S$ and $f$ is non-negative, then $\int c \cdot f \mathrm{~d} M=$ $c \cdot \int^{\prime} f \mathrm{~d} M$.

(60) Suppose $f$ is simple function in $S$ and $f$ is non-positive. Then

(i) $\int c \cdot f \mathrm{~d} M=-c \cdot \int^{\prime}-f \mathrm{~d} M$, and

(ii) $\int c \cdot f \mathrm{~d} M=-\left(c \cdot \int^{\prime}-f \mathrm{~d} M\right)$.

The theorem is a consequence of (35), (30), and (59).

(61) Let us consider a non empty set $X$, a $\sigma$-field $S$ of subsets of $X$, a $\sigma$ measure $M$ on $S$, and a partial function $f$ from $X$ to $\overline{\mathbb{R}}$. Suppose there exists an element $A$ of $S$ such that $A=\operatorname{dom} f$ and $f$ is measurable on $A$ and $f$ is non-positive. Then $0 \geqslant \int f \mathrm{~d} M$. The theorem is a consequence of (57).

(62) Let us consider a non empty set $X$, a $\sigma$-field $S$ of subsets of $X$, a $\sigma$ measure $M$ on $S$, a partial function $f$ from $X$ to $\overline{\mathbb{R}}$, and elements $A, B, E$ of $S$. Suppose $E=\operatorname{dom} f$ and $f$ is measurable on $E$ and $f$ is non-positive and $A$ misses $B$. Then $\int f\left\lceil(A \cup B) \mathrm{d} M=\int f\left\lceil A \mathrm{~d} M+\int f\lceil B \mathrm{~d} M\right.\right.$. The theorem is a consequence of (3) and (52).

(63) Let us consider a non empty set $X$, a $\sigma$-field $S$ of subsets of $X$, a $\sigma$ measure $M$ on $S$, a partial function $f$ from $X$ to $\overline{\mathbb{R}}$, and elements $A, E$ of $S$. Suppose $E=\operatorname{dom} f$ and $f$ is measurable on $E$ and $f$ is non-positive. Then $0 \geqslant \int f\lceil A \mathrm{~d} M$. The theorem is a consequence of (61) and (1).

(64) Let us consider a non empty set $X$, a $\sigma$-field $S$ of subsets of $X$, a $\sigma$ measure $M$ on $S$, a partial function $f$ from $X$ to $\overline{\mathbb{R}}$, and elements $A, B, E$ of $S$. Suppose $E=\operatorname{dom} f$ and $f$ is measurable on $E$ and $f$ is non-positive and $A \subseteq B$. Then $\int f\left\lceil A \mathrm{~d} M \geqslant \int f\lceil B \mathrm{~d} M\right.$. The theorem is a consequence of (3) and (52). 
3. Convergence Theorems for Non Positive Function's Integration

Now we state the propositions:

(65) Let us consider a non empty set $X$, a $\sigma$-field $S$ of subsets of $X$, a $\sigma$ measure $M$ on $S$, an element $E$ of $S$, and a partial function $f$ from $X$ to $\overline{\mathbb{R}}$. Suppose $E=\operatorname{dom} f$ and $f$ is measurable on $E$ and $f$ is non-positive and $M(E \cap \mathrm{EQ}-\operatorname{dom}(f,-\infty)) \neq 0$. Then $\int f \mathrm{~d} M=-\infty$. The theorem is a consequence of (9) and (52).

(66) Let us consider a non empty set $X$, a $\sigma$-field $S$ of subsets of $X$, a $\sigma$ measure $M$ on $S$, an element $E$ of $S$, and partial functions $f, g$ from $X$ to $\overline{\mathbb{R}}$. Suppose $E \subseteq \operatorname{dom} f$ and $E \subseteq \operatorname{dom} g$ and $f$ is measurable on $E$ and $g$ is measurable on $E$ and $f$ is non-positive and for every element $x$ of $X$ such that $x \in E$ holds $g(x) \leqslant f(x)$. Then $\int g\left\lceil E \mathrm{~d} M \leqslant \int f\lceil E \mathrm{~d} M\right.$. The theorem is a consequence of (3) and (52).

(67) Let us consider a non empty set $X$, a sequence $F$ of partial functions from $X$ into $\overline{\mathbb{R}}$, a $\sigma$-field $S$ of subsets of $X$, an element $E$ of $S$, and a natural number $m$. Suppose $F$ has the same dom and $E=\operatorname{dom}(F(0))$ and for every natural number $n, F(n)$ is measurable on $E$ and $F(n)$ is without $+\infty$. Then $\left(\sum_{\alpha=0}^{\kappa} F(\alpha)\right)_{\kappa \in \mathbb{N}}(m)$ is measurable on $E$. The theorem is a consequence of (37), (42), and (46).

(68) Let us consider a non empty set $X$, a $\sigma$-field $S$ of subsets of $X$, a $\sigma$ measure $M$ on $S$, a sequence $F$ of partial functions from $X$ into $\overline{\mathbb{R}}$, an element $E$ of $S$, a sequence $I$ of extended reals, and a natural number $m$. Suppose $E=\operatorname{dom}(F(0))$ and $F$ is additive and has the same dom and for every natural number $n, F(n)$ is measurable on $E$ and $F(n)$ is non-positive and $I(n)=\int F(n) \mathrm{d} M$. Then $\int\left(\sum_{\alpha=0}^{\kappa} F(\alpha)\right)_{\kappa \in \mathbb{N}}(m) \mathrm{d} M=$ $\left(\sum_{\alpha=0}^{\kappa} I(\alpha)\right)_{\kappa \in \mathbb{N}}(m)$.

Proof: Set $G=-F$. Set $J=-I . G(0)=-F(0)$. $G$ has the same dom. For every natural number $n, F(n)$ is measurable on $E$ and $F(n)$ is without $+\infty$. For every natural number $n, G(n)$ is measurable on $E$ and $G(n)$ is non-negative and $J(n)=\int G(n) \mathrm{d} M \cdot \int\left(\sum_{\alpha=0}^{\kappa} G(\alpha)\right)_{\kappa \in \mathbb{N}}(m) \mathrm{d} M=$ $\left(\sum_{\alpha=0}^{\kappa} J(\alpha)\right)_{\kappa \in \mathbb{N}}(m) \cdot \int\left(-\left(\sum_{\alpha=0}^{\kappa} F(\alpha)\right)_{\kappa \in \mathbb{N}}\right)(m) \mathrm{d} M=\left(\sum_{\alpha=0}^{\kappa} J(\alpha)\right)_{\kappa \in \mathbb{N}}(m)$. $\int\left(-\left(\sum_{\alpha=0}^{\kappa} F(\alpha)\right)_{\kappa \in \mathbb{N}}\right)(m) \mathrm{d} M=-\left(\sum_{\alpha=0}^{\kappa} I(\alpha)\right)_{\kappa \in \mathbb{N}}(m)$.

$\int-\left(\sum_{\alpha=0}^{\kappa} F(\alpha)\right)_{\kappa \in \mathbb{N}}(m) \mathrm{d} M=-\left(\sum_{\alpha=0}^{\kappa} I(\alpha)\right)_{\kappa \in \mathbb{N}}(m)$. $-\int\left(\sum_{\alpha=0}^{\kappa} F(\alpha)\right)_{\kappa \in \mathbb{N}}(m) \mathrm{d} M=-\left(\sum_{\alpha=0}^{\kappa} I(\alpha)\right)_{\kappa \in \mathbb{N}}(m)$.

(69) Let us consider a non empty set $X$, a $\sigma$-field $S$ of subsets of $X$, a $\sigma$ measure $M$ on $S$, a sequence $F$ of partial functions from $X$ into $\overline{\mathbb{R}}$, an element $E$ of $S$, and a partial function $f$ from $X$ to $\overline{\mathbb{R}}$. Suppose $E \subseteq \operatorname{dom} f$ and $f$ is non-positive and $f$ is measurable on $E$ and for every natural 
number $n, F(n)$ is simple function in $S$ and $F(n)$ is non-positive and $E \subseteq \operatorname{dom}(F(n))$ and for every element $x$ of $X$ such that $x \in E$ holds $F \# x$ is summable and $f(x)=\sum(F \# x)$. Then there exists a sequence $I$ of extended reals such that

(i) for every natural number $n, I(n)=\int F(n)\lceil E \mathrm{~d} M$, and

(ii) $I$ is summable, and

(iii) $\int f\left\lceil E \mathrm{~d} M=\sum I\right.$.

Proof: Set $g=-f$. Set $G=-F$. $G$ is additive. For every natural number $n, G(n)$ is simple function in $S$ and $G(n)$ is non-negative and $E \subseteq \operatorname{dom}(G(n))$. For every element $x$ of $X$ such that $x \in E$ holds $G \# x$ is summable and $g(x)=\sum(G \# x)$. Consider $J$ being a sequence of extended reals such that for every natural number $n, J(n)=\int G(n)\lceil E \mathrm{~d} M$ and $J$ is summable and $\int g\left\lceil E \mathrm{~d} M=\sum J\right.$. For every natural number $n$, $I(n)=\int F(n)\left\lceil E \mathrm{~d} M \cdot \int g\left\lceil E \mathrm{~d} M=-\int f\left\lceil E \mathrm{~d} M \cdot \lim \left(\sum_{\alpha=0}^{\kappa} I(\alpha)\right)_{\kappa \in \mathbb{N}}=\right.\right.\right.$ $-\int g\lceil E \mathrm{~d} M$.

(70) Let us consider a non empty set $X$, a $\sigma$-field $S$ of subsets of $X$, a $\sigma$ measure $M$ on $S$, an element $E$ of $S$, and a partial function $f$ from $X$ to $\overline{\mathbb{R}}$. Suppose $E \subseteq \operatorname{dom} f$ and $f$ is non-positive and $f$ is measurable on $E$. Then there exists a sequence $F$ of partial functions from $X$ into $\overline{\mathbb{R}}$ such that

(i) $F$ is additive, and

(ii) for every natural number $n, F(n)$ is simple function in $S$ and $F(n)$ is non-positive and $F(n)$ is measurable on $E$, and

(iii) for every element $x$ of $X$ such that $x \in E$ holds $F \# x$ is summable and $f(x)=\sum(F \# x)$, and

(iv) there exists a sequence $I$ of extended reals such that for every natural number $n, I(n)=\int F(n)\left\lceil E \mathrm{~d} M\right.$ and $I$ is summable and $\int f\lceil E \mathrm{~d} M=$ $\sum I$.

Proof: Set $g=-f$. Consider $G$ being a sequence of partial functions from $X$ into $\overline{\mathbb{R}}$ such that $G$ is additive and for every natural number $n, G(n)$ is simple function in $S$ and $G(n)$ is non-negative and $G(n)$ is measurable on $E$ and for every element $x$ of $X$ such that $x \in E$ holds $G \# x$ is summable and $g(x)=\sum(G \# x)$ and there exists a sequence $J$ of extended reals such that for every natural number $n, J(n)=\int G(n)\lceil E \mathrm{~d} M$ and $J$ is summable and $\int g\left\lceil E \mathrm{~d} M=\sum J\right.$. For every natural number $n, F(n)$ is simple function in $S$ and $F(n)$ is non-positive and $F(n)$ is measurable on $E$. For every element $x$ of $X$ such that $x \in E$ holds $F \# x$ is summable and $f(x)=\sum(F \# x)$. There exists a sequence $I$ of extended reals such that 
for every natural number $n, I(n)=\int F(n)\lceil E \mathrm{~d} M$ and $I$ is summable and $\int f\left\lceil E \mathrm{~d} M=\sum I\right.$.

Let us consider a non empty set $X$, a $\sigma$-field $S$ of subsets of $X$, a $\sigma$-measure $M$ on $S$, a sequence $F$ of partial functions from $X$ into $\overline{\mathbb{R}}$, and an element $E$ of $S$. Now we state the propositions:

(71) Suppose $E=\operatorname{dom}(F(0))$ and $F$ has the same dom and for every natural number $n, F(n)$ is non-positive and $F(n)$ is measurable on $E$. Then there exists a sequence $F_{1}$ of $(X \dot{\rightarrow} \overline{\mathbb{R}})^{\mathbb{N}}$ such that for every natural number $n$, for every natural number $m, F_{1}(n)(m)$ is simple function in $S$ and $\operatorname{dom}\left(F_{1}(n)(m)\right)=\operatorname{dom}(F(n))$ and for every natural number $m, F_{1}(n)(m)$ is non-positive and for every natural numbers $j, k$ such that $j \leqslant k$ for every element $x$ of $X$ such that $x \in \operatorname{dom}(F(n))$ holds $F_{1}(n)(j)(x) \geqslant F_{1}(n)(k)(x)$ and for every element $x$ of $X$ such that $x \in \operatorname{dom}(F(n))$ holds $F_{1}(n) \# x$ is convergent and $\lim \left(F_{1}(n) \# x\right)=F(n)(x)$.

Proof: Define $\mathcal{Q}$ [element of $\mathbb{N}$, set] $\equiv$ for every sequence $G$ of partial functions from $X$ into $\overline{\mathbb{R}}$ such that $\$_{2}=G$ holds for every natural number $m, G(m)$ is simple function in $S$ and $\operatorname{dom}(G(m))=\operatorname{dom}\left(F\left(\$_{1}\right)\right)$ and for every natural number $m, G(m)$ is non-positive and for every natural numbers $j, k$ such that $j \leqslant k$ for every element $x$ of $X$ such that $x \in$ $\operatorname{dom}\left(F\left(\$_{1}\right)\right)$ holds $G(j)(x) \geqslant G(k)(x)$ and for every element $x$ of $X$ such that $x \in \operatorname{dom}\left(F\left(\$_{1}\right)\right)$ holds $G \# x$ is convergent and $\lim (G \# x)=F\left(\$_{1}\right)(x)$. For every element $n$ of $\mathbb{N}$, there exists a sequence $G$ of partial functions from $X$ into $\overline{\mathbb{R}}$ such that for every natural number $m, G(m)$ is simple function in $S$ and $\operatorname{dom}(G(m))=\operatorname{dom}(F(n))$ and for every natural number $m, G(m)$ is non-positive and for every natural numbers $j, k$ such that $j \leqslant k$ for every element $x$ of $X$ such that $x \in \operatorname{dom}(F(n))$ holds $G(j)(x) \geqslant$ $G(k)(x)$ and for every element $x$ of $X$ such that $x \in \operatorname{dom}(F(n))$ holds $G \# x$ is convergent and $\lim (G \# x)=F(n)(x)$. For every element $n$ of $\mathbb{N}$, there exists an element $G$ of $(X \dot{\bar{R}})^{\mathbb{N}}$ such that $\mathcal{Q}[n, G]$. Consider $F_{1}$ being a sequence of $(X \dot{\rightarrow} \overline{\mathbb{R}})^{\mathbb{N}}$ such that for every element $n$ of $\mathbb{N}, \mathcal{Q}\left[n, F_{1}(n)\right]$. For every natural number $n$, for every natural number $m, F_{1}(n)(m)$ is simple function in $S$ and $\operatorname{dom}\left(F_{1}(n)(m)\right)=\operatorname{dom}(F(n))$ and for every natural number $m, F_{1}(n)(m)$ is non-positive and for every natural numbers $j, k$ such that $j \leqslant k$ for every element $x$ of $X$ such that $x \in \operatorname{dom}(F(n))$ holds $F_{1}(n)(j)(x) \geqslant F_{1}(n)(k)(x)$ and for every element $x$ of $X$ such that $x \in$ $\operatorname{dom}(F(n))$ holds $F_{1}(n) \# x$ is convergent and $\lim \left(F_{1}(n) \# x\right)=F(n)(x)$.

(72) Suppose $E=\operatorname{dom}(F(0))$ and $F$ is additive and has the same dom and for every natural number $n, F(n)$ is measurable on $E$ and $F(n)$ is nonpositive. Then there exists a sequence $I$ of extended reals such that for every natural number $n, I(n)=\int F(n) \mathrm{d} M$ and $\int\left(\sum_{\alpha=0}^{\kappa} F(\alpha)\right)_{\kappa \in \mathbb{N}}(n) \mathrm{d} M=$ 
$\left(\sum_{\alpha=0}^{\kappa} I(\alpha)\right)_{\kappa \in \mathbb{N}}(n)$.

Proof: Set $G=-F . G(0)=-F(0)$. $G$ has the same dom. For every natural number $n, G(n)$ is measurable on $E$ and $G(n)$ is non-negative. Consider $J$ being a sequence of extended reals such that for every natural number $n$, $J(n)=\int G(n) \mathrm{d} M$ and $\int\left(\sum_{\alpha=0}^{\kappa} G(\alpha)\right)_{\kappa \in \mathbb{N}}(n) \mathrm{d} M=\left(\sum_{\alpha=0}^{\kappa} J(\alpha)\right)_{\kappa \in \mathbb{N}}(n)$. For every natural number $n, F(n)$ is measurable on $E$ and $F(n)$ is without $+\infty$.

(73) Suppose $E \subseteq \operatorname{dom}(F(0))$ and $F$ is additive and has the same dom and for every natural number $n, F(n)$ is non-positive and $F(n)$ is measurable on $E$ and for every element $x$ of $X$ such that $x \in E$ holds $F \# x$ is summable. Then there exists a sequence $I$ of extended reals such that

(i) for every natural number $n, I(n)=\int F(n)\lceil E \mathrm{~d} M$, and

(ii) $I$ is summable, and

(iii) $\int \lim \left(\sum_{\alpha=0}^{\kappa} F(\alpha)\right)_{\kappa \in \mathbb{N}}\left\lceil E \mathrm{~d} M=\sum I\right.$.

Proof: Set $G=-F . G(0)=-F(0)$. $G$ is additive. $G$ has the same dom. For every natural number $n, G(n)$ is non-negative and $G(n)$ is measurable on $E$. For every element $x$ of $X$ such that $x \in E$ holds $G \# x$ is summable. Consider $J$ being a sequence of extended reals such that for every natural number $n, J(n)=\int G(n)\lceil E \mathrm{~d} M$ and $J$ is summable and $\int \lim \left(\sum_{\alpha=0}^{\kappa} G(\alpha)\right)_{\kappa \in \mathbb{N}} \uparrow E \mathrm{~d} M=\sum J$. For every natural number $n, I(n)=$ $\int F(n)\left\lceil E \mathrm{~d} M\right.$. Define $\mathcal{H}$ (natural number) $=F\left(\$_{1}\right)\lceil E$. Consider $H$ being a sequence of partial functions from $X$ into $\overline{\mathbb{R}}$ such that for every natural number $n, H(n)=\mathcal{H}(n) \cdot \lim \left(\sum_{\alpha=0}^{\kappa} H(\alpha)\right)_{\kappa \in \mathbb{N}}=\lim \left(\sum_{\alpha=0}^{\kappa} F(\alpha)\right)_{\kappa \in \mathbb{N}}\lceil E$. Define $\mathcal{K}$ (natural number) $=G\left(\$_{1}\right)\lceil E$. Consider $K$ being a sequence of partial functions from $X$ into $\overline{\mathbb{R}}$ such that for every natural number $n$, $K(n)=\mathcal{K}(n) \cdot \lim \left(\sum_{\alpha=0}^{\kappa} K(\alpha)\right)_{\kappa \in \mathbb{N}}=\lim \left(\sum_{\alpha=0}^{\kappa} G(\alpha)\right)_{\kappa \in \mathbb{N}}\lceil E$. For every element $n$ of $\mathbb{N}, H(n)=(-K)(n) \cdot \lim \left(\sum_{\alpha=0}^{\kappa} H(\alpha)\right)_{\kappa \in \mathbb{N}}=$ $-\lim \left(\sum_{\alpha=0}^{\kappa} K(\alpha)\right)_{\kappa \in \mathbb{N}}$. For every natural number $n, K(n)$ is measurable on $E$ and $K(n)$ is without $-\infty \cdot \int\left(-\lim \left(\sum_{\alpha=0}^{\kappa} K(\alpha)\right)_{\kappa \in \mathbb{N}}\right)\lceil E \mathrm{~d} M=$ $-\int \lim \left(\sum_{\alpha=0}^{\kappa} K(\alpha)\right)_{\kappa \in \mathbb{N}}\lceil E \mathrm{~d} M$.

(74) Suppose $E=\operatorname{dom}(F(0))$ and $F(0)$ is non-positive and $F$ has the same dom and for every natural number $n, F(n)$ is measurable on $E$ and for every natural numbers $n, m$ such that $n \leqslant m$ for every element $x$ of $X$ such that $x \in E$ holds $F(n)(x) \geqslant F(m)(x)$ and for every element $x$ of $X$ such that $x \in E$ holds $F \# x$ is convergent. Then there exists a sequence $I$ of extended reals such that

(i) for every natural number $n, I(n)=\int F(n) \mathrm{d} M$, and

(ii) $I$ is convergent, and 
(iii) $\int \lim F \mathrm{~d} M=\lim I$.

Proof: Set $G=-F . G(0)=-F(0)$. For every natural number $n, G(n)$ is measurable on $E$ by [4, (63)], (37). For every natural numbers $n, m$ such that $n \leqslant m$ for every element $x$ of $X$ such that $x \in E$ holds $G(n)(x) \leqslant G(m)(x)$. For every element $x$ of $X$ such that $x \in E$ holds $G \# x$ is convergent. Consider $J$ being a sequence of extended reals such that for every natural number $n, J(n)=\int G(n) \mathrm{d} M$ and $J$ is convergent and $\int \lim G \mathrm{~d} M=\lim J$. Set $I=-J$. For every natural number $n, I(n)=$ $\int F(n) \mathrm{d} M$. For every element $x$ of $X$ such that $x \in \operatorname{dom} \lim G$ holds $(\lim G)(x)=(-\lim F)(x)$ by $(38),[3,(17)] . \int \lim G \mathrm{~d} M=-\int \lim F \mathrm{~d} M$.

\section{REFERENCES}

[1] Grzegorz Bancerek, Czesław Byliński, Adam Grabowski, Artur Korniłowicz, Roman Matuszewski, Adam Naumowicz, Karol Pąk, and Josef Urban. Mizar: State-of-the-art and beyond In Manfred Kerber, Jacques Carette, Cezary Kaliszyk, Florian Rabe, and Volker Sorge, editors, Intelligent Computer Mathematics, volume 9150 of Lecture Notes in Computer Science, pages 261-279. Springer International Publishing, 2015. ISBN 978-3319-20614-1. doi $10.1007 / 978-3-319-20615-8 \_17$.

[2] Vladimir Igorevich Bogachev and Maria Aparecida Soares Ruas. Measure theory, volume 1. Springer, 2007.

[3] Noboru Endou. Extended real-valued double sequence and its convergence. Formalized Mathematics, 23(3):253-277, 2015. doi 10.1515/forma-2015-0021.

[4] Noboru Endou. Fubini's theorem on measure. Formalized Mathematics, 25(1):1-29, 2017. doi:10.1515/forma-2017-0001.

[5] Noboru Endou and Yasunari Shidama. Integral of measurable function. Formalized Mathematics, 14(2):53-70, 2006. doi 10.2478/v10037-006-0008-x.

[6] Noboru Endou, Keiko Narita, and Yasunari Shidama. The Lebesgue monotone convergence theorem. Formalized Mathematics, 16(2):167-175, 2008. doi:10.2478/v10037-008-0023-1.

[7] Adam Grabowski, Artur Korniłowicz, and Adam Naumowicz. Four decades of Mizar. Journal of Automated Reasoning, 55(3):191-198, 2015. doi 10.1007/s10817-015-9345-1

[8] P. R. Halmos. Measure Theory. Springer-Verlag, 1974.

Received September 3, 2017

The English version of this volume of Formalized Mathematics was financed under agreement 548/P-DUN/2016 with the funds from the Polish Minister of Science and Higher Education for the dissemination of science. 